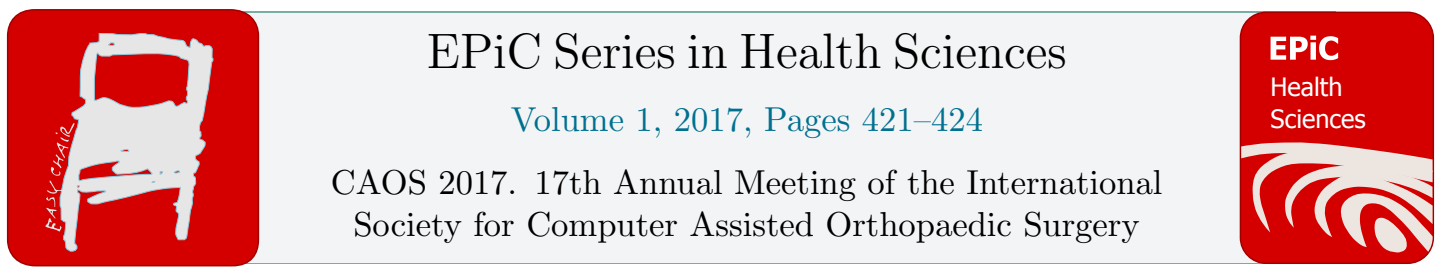

\title{
Robotic assisted fixation of sacral fractures - initial experience
}

\author{
Yoram A. Weil ${ }^{1}$, MD, Josh E Schroeder MD, Rami Mosheiff MD, Meir Liebergall, MD, Amal \\ Khoury MD \\ ${ }^{1}$ Department of Orthopaedics, Hadassah Hebrew University Hospital, Jerusalem, Israel \\ POB 12000, Jeursaelm 91120 email - weily@,hadassah.org.il; yoramweil@gmail.com
}

\begin{abstract}
Unstable sacral fractures are challenging for orthopaedic trauma surgeons. In most cases percutaneous fixation techniques are utilized after reduction. However, these techniuqes are not risk free mainly due to anatomical considerations. Screw misplacement is quite common and concerning. As spine surgery evolved, a miniature robotic guidance system was successfully utilized in pedicular screw insertion. The aim of the study was to demonstrate th use of the miniature robot in the fixation of unstable sacral fracutres.

Patients and Methods: 9 patients with unstable sacral fracutres without significant displacement were eligible for percutaneous fixation. These included 7 traumatic fractures and 2 pathological fractures. All fixation constructs were planned using a preoperative CT scans. The patients were placed prone and the robot was mounted on a Dynamic Reference Bridge (DRB) and a 2 verification fluoroscopic images were taken. The robot was mounted on the DRB and was sent by the computer to point to the desired screw(s) trajectory. The guide wires were inserted through stab wounds and screws were placed subsequently. CT scans were made postoperatively and fluoroscopic and operative time were recorded intraoperatively. Results: Mean patient age was 29 (17-63) number of screws ranged 1-6 (average 2). Mean operative time was $50 \mathrm{~min}$ (range 15-90), and average fluoroscopic time was $18 \mathrm{sec}$ (7-42). None was the screw was misplaced. Conclusion: Robotic assisted fixation of sacral fracture is promising. At this time it is limited to nondisplaced fractures.
\end{abstract}

\section{Introduction}

Unstable sacral fractures are a challenge for the orthopedic trauma surgeons. Vertical instability and comminution are often encountered and may compromise fixation success.

In most cases open or closed reduction is required and many surgeons today prefer percutaneous fixation techniques, using image guidance [1].

Several fixation modalities have been proposed including iliosacral screws, transiliac, transsacral screws and lumbopelvic fixation, as well as combinations of all the above. However, this 
technique is not risk free, mainly due to anatomical considerations. The anatomical safe corridor which allows for the fixation of the ilium to the first and second sacral vertebrae can be narrow, limited by exiting nerve root anteriorly, neural foramina caudally and sacral canal posteriorly. Furthermore the size and shape of the safe corridor is anatomically varied among individuals.

Although this procedure is very common, the complication rates are not as low as can be expected or previously described. Some studies reports screw misplacement rates between $13 \%$ to $30 \%[2]$.

As spine surgery involves similar high risk fixation, a miniature hexapod robotic device has been introduced to improve the accuracy of pedicular screw placement [3]. This is being done using a preoperative $\mathrm{CT}$, intraoperative registration using CT to fluoroscopic merging algorithms and final implant placement by the robot based on the preoperative planning. The aim of this study is demonstrating the use of the miniature robot in the fixation of unstable sacral fractures

\section{Patients and methods}

Nine patients were treated with robotic assisted iliosacral and lumbopelvic fixation between 2013-2016. These included seven traumatic fractures, one insufficiency sacral fractures and one pathological fractures. Fixation included iliosacral screws alone (4 cases) and lumbopelvic fixation coupled with iliosacral screw- commonly known as triangular fixation (5 cases). Surgical technique: A basic requirement was that the sacral fracture was minimally displaced prior to surgery. A CT scan was performed preoperatively and directed to the robot's workstation. The screws were preoperative planned according the surgeon preference and fracture configuration (Fig1a). Patients were placed prone on a radiolucent table. A plastic Dynamic Reference Bridge (DRB) was mounted on the table connected to a pin placed one a lumbar vertebra spinous process. Intraoperative registration of 2 fluoroscopic images with at least 60 degrees of angle was necessary (fig $1 \mathrm{~b}$ ). The robot was mounted on the DRB and was sent to the required trajectories. A percutaneous incision was performed and either a drill (pedicle screw) or a $2.8 \mathrm{~mm}$ guidewire (iliosacral screw) was introduced. After all guides were placed, verification radiographs were placed. Screws were placed over the guidewires/drill-bits using the standard surgical technique. Fixation was completed and postoperative care was given depending on the fracture type. All patients underwent postoperative CT for verification of implant placement.

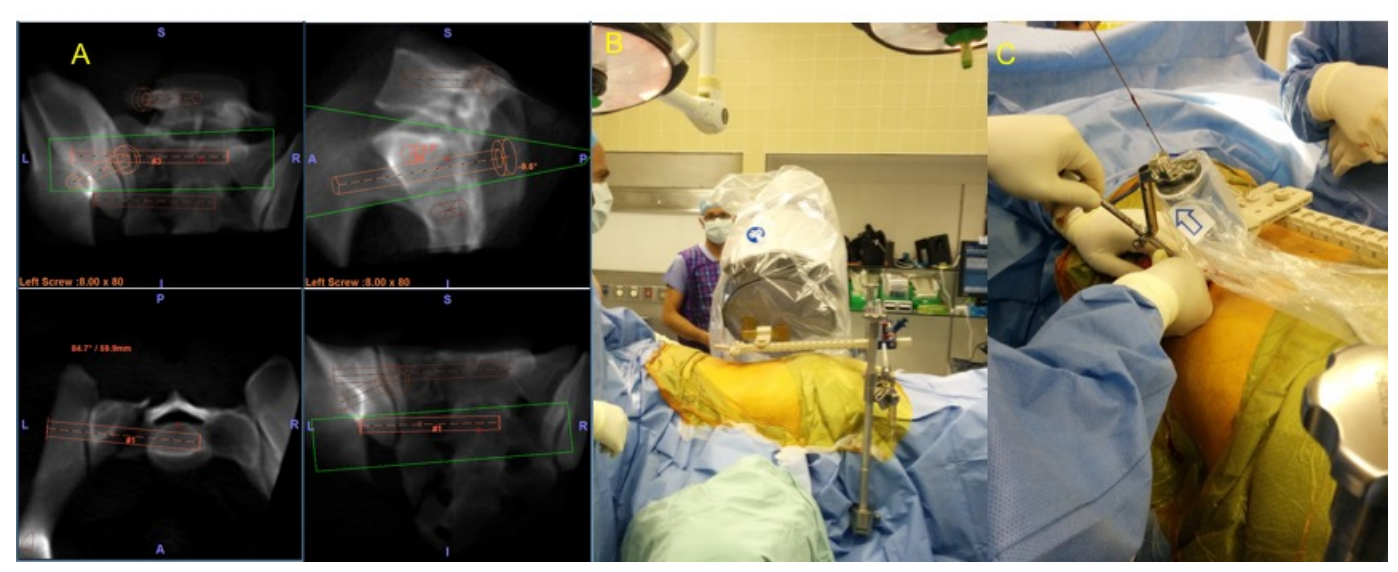


figure 1: (A) preoperative planning of lumbopelvic fixation of an unstable sacral fracture (B) intraoperative registration with the DRB mounted (C) insertion of the guidewire using the robot.

\section{Results}

Mean patient age was 29 (range 17-63). Fractures type included traumatic fractures due to motor vehicle accidents and falls ( 7 patients), 1 insufficiency fracture and one pathological fractures. Number of screws were between 1 (iliosacral) to 6 (lumbopelvic) (average 2). Operative time ranged from 15 to 90 minutes (average $50 \mathrm{~min}$ ),. Average fluoroscopic time was 18 seconds (range 7 to 42 sec). All CT demonstrated complete intraosseous trajectory of the screws without breaching (Fig 2)

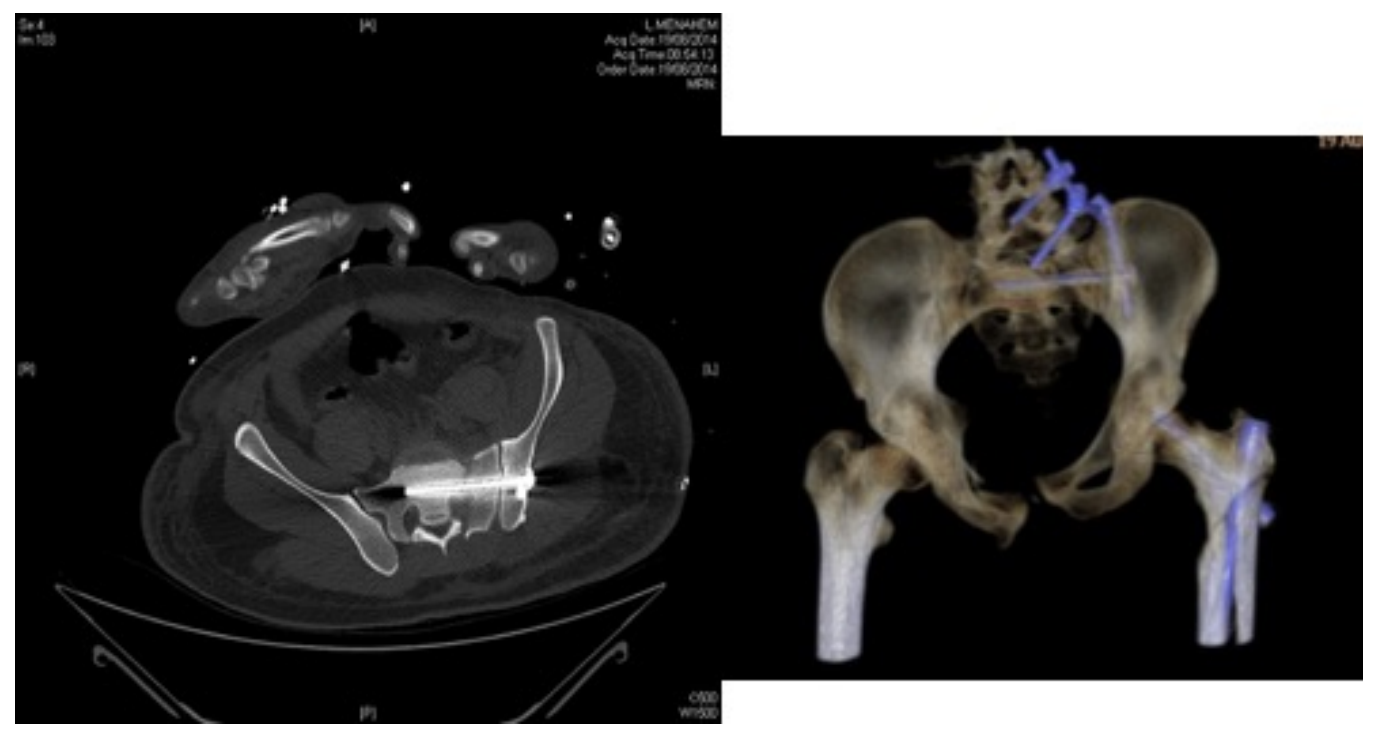

Figure 2 - an axial and 3D CT demonstrating intraosseous position of all fixation consturcts.

\section{Discussion}

The role for minimally invasive fixation of posterior pelvic ring injuries is now well established. While surgical reduction is still a challenge, the adequate, precise and quick placement of iliosacral screws can become an uneasy task even among experienced surgeons. We demonstrate a high degree of success using robotic guidance for placing iliosacral with or without lumbar and iliac screws in unstable sacral fractures. We achieved a high degree of accuracy with limiting the radiation time to a minimum. As the quoted fluoroscopic time for an average iliosacral screw placement can be of minutes [4] we are encouraged by the relatively low radiation obtained with this system. Also, the time for setup and activation of the robot was negligible as seen in the net operative timings. However , it should be borne in mind that our center is very familiar with the application of this system in spine surgery, and a dedicated team was present in all cases.

A Major limitation in this technique that it requires the fracture to be either minimally displaced or reduced before surgery. Therefore, any changes in the anatomy such as fracture reduction 
cannot be made prior to screw insertion. A possible solution might be an intraoperative CT registration such as currently done is some experimental hybrid rooms [5] - yet this has still to be developed.

\section{References:}

1. Routt ML, Jr., Kregor PJ, Simonian PT, Mayo KA. Early results of percutaneous iliosacral screws placed with the patient in the supine position. J Orthop Trauma. 1995;9(3):207-214.

2. Herman A, Keener E, Dubose C, Lowe JA. Simple mathematical model of sacroiliac screws safe-zone-Easy to implement by pelvic inlet and outlet views. J Orthop Res. 2016.

3. Hu X, Ohnmeiss DD, Lieberman IH. Robotic-assisted pedicle screw placement: lessons learned from the first 102 patients. Eur Spine J. 2013;22(3):661-666.

4. Collinge C, Coons D, Tornetta P, Aschenbrenner J. Standard multiplanar fluoroscopy versus a fluoroscopically based navigation system for the percutaneous insertion of iliosacral screws: a cadaver model. J Orthop Trauma. 2005;19(4):254-258.

5. Richter PH, Gebhard F, Dehner C, Scola A. Accuracy of computer-assisted iliosacral screw placement using a hybrid operating room. Injury. 2016;47(2):402-407. 